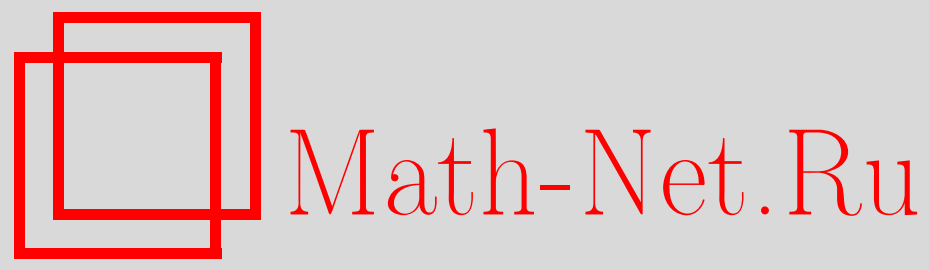

В. М. Закалюкин, О. М. Мясниченко, Лагранжевы особенности при симплектической редукции, Функи. анализ и его прил., 1998, том 32, выпуск $1,1-11$

DOI: https://doi.org/10.4213/faa393

Использование Общероссийского математического портала MathNet.Ru подразумевает, что вы прочитали и согласны с пользовательским соглашением http://www.mathnet.ru/rus/agreement

Параметры загрузки:

IP: 34.229 .108 .108

26 апреля 2023 г., 09:57:53

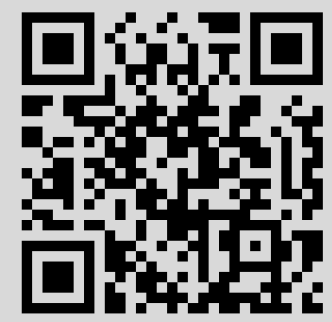


Функииональньй анализ и еәо приложения

1998, т. 32, вып. 1, с. 1-11

УДК 514.853

\section{Лагранжевы особенности при симплектической редукции}

(c) 1998. В. М. ЗАКАЛЮКИн, О. М. МяСНиЧЕНко

\section{Введение}

Лагранжевы многообразия с особенностями встречаются во многих задачах анализа. Впервые интересный класс таких многообразий (раскрытые ласточкины хвосты) возник в вариационной задаче с односторонними ограничениями (задаче об обходе препятствия) [1]. Волновые фронты простых устойчивых лагранжевых проектирований раскрытых ласточкиных хвостов тесно связаны с дискриминантами коксетеровских групп, порожденных отражениями $[2,3]$.

Решения задачи Коши для уравнения Гамильтона-Якоби с начальными условиями, образующими изотропное подмногообразие гиперповерхности уровня гамильтониана, приводят к другим классам особых лагранжевых многообразий - раскрытым зонтикам Уитни $[4,3]$.

В этих примерах особые лагранжевы многообразия порождаются (иногда с дополнительными компонентами) неморсовскими производящими семействами, т.е. являются образами при симплектической редукции неособого лагранжева подмногообразия большей размерности относительно нетрансверсальной ему гиперповерхности. Условия нетрансверсальности в этих ситуациях весьма специфичны - пересечение лагранжева и коизотропного подмногообразий содержит неособое изотропное подмногообразие, имеющее коразмерность 1 в лагранжевом. Раскрытые ласточкины хвосты и зонтики Уитни имеют (за исключением случая минимальных размерностей) неизолированные особенности.

Естественной группой эквивалентностей в таких задачах является группа симплектоморфизмов, сохраняющих некоторое лагранжево расслоение (например, кокасательное). При этом устойчивость таких особенностей рассматривается в классе лагранжевых вложений заданного особого подмногообразия.

Общие конструкции, позволяющие получать устойчивые простые (т.е. не содержащие непрерывных инвариантов) ростки лагранжевых проектирований для такой группы, описаны в [5].

В настоящей работе мы классифицируем простые лагранжевы ростки, получаемые в результате произвольной симплектической редукции, с точностью до действия большей группы - группы всех симплектоморфизмов.

Отметим, что похожая задача об особенностях симплектической редукции

\footnotetext{
^ Работа выполнена при поддержке грантов РФФИ 96-01-00710 и ИНТАС 96-0713.
} 
(правда, для группы симплектоморфизмов, сохраняющих расслоение) рассмотрена в [6].

Наш список простых классов весьма поучителен - появляется еше одна новая связь простых алгебр Ли $A, B, C, D, E, F$ с лагранжевыми особенностями.

Простейшую особенность, отвечающую морсовской нетрансверсальности, мы рассматриваем более подробно. Ее устойчивость доказана по отношению к меньшей группе, состоящей из симплектоморфизмов, сохраняющих расслоение объемлющего симплектического пространства, одним из слоев которого является заданная гиперповерхность.

Эта особенность используется для оценки числа траекторий натуральной механической системы, стартующих с заданного подмногообразия конфигурационного пространства и выходящих на границу области возможных движений.

\section{Определения и результаты}

Рассмотрим пару $\left(L^{n}, C^{2 n-k}\right)$, состоящую из ростков в нуле лагранжева подмногообразия $L$ и коизотропного подмногообразия $C$ стандартного симплектического пространства $\left(\mathbb{R}^{2 n}, \omega\right)$. Две такие пары назовем эквивалентными, если одна переводится в другую некоторым локальным симплектоморфизмом окрестности нуля пространства $\mathbb{R}^{2 n}$.

Паре $(L, C)$ отвечает росток редуцированного лагранжева подмногообразия (вообще говоря, особого) $\rho(L, C)$ в симплектической базе расслоения многообразия $C$ на характеристические слои (на которых симплектическая форма является нулевой).

Пара называется простой, если ростки $L$ и $C$ имеют такие представители, ростки малых деформаций которых во всякой точке задают пары, эквивалентные одной из конечного списка нормальных форм.

Хорошо известно [7], что если $L^{n}$ трансверсально в нуле ростку многообразия $C^{2 n-k}$, то пересечение $L \cap C$ трансверсально характеристическому слоению многообразия $C$ и $\rho(L, C)$ - неособое лагранжево подмногообразие. Все такие пары эквивалентны между собой.

Надстройкой ранга $m$ пары $(L, C)$ назовем пару $(L \times X, C \times Y)$ в симплектическом пространстве $\mathbb{R}^{2 n} \times \mathbb{R}^{2 m}$, снабженном симплектической формой, равной сумме стандартных симплектических форм на сомножителях, поднятых на произведение с помошью соответствующих проекций. 3 десь $X$ и $Y$ - трансверсальные между собой лагранжевы подмногообразия в $\mathbb{R}^{2 m}$. Очевидно, что все надстройки данного ранга эквивалентных пар эквивалентны между собой.

Всякая нетрансверсальная пара эквивалентна надстройке (касательной) пары, для которой лагранжево подмногообразие касается коизотропного. В этом последнем случае существуют координаты Дарбу $(p, q)$ с началом в рассматриваемой точке, в которых $C$ задается уравнениями $p_{1}=\cdots=$ $p_{k}=0$ и росток $L$ задается некоторой производящей функцией $S(q)$,

$$
L=\left\{(p, q) \mid p=\frac{\partial S}{\partial q}\right\}
$$


принадлежащей кубу максимального идеала $\mathbf{m}_{q}$ в кольце ростков в нуле функций от $q$. Такие координаты Дарбу будем называть приспособленнылми, а такую производящую функцию $S$ - приведенной.

Приспособленные координаты (а следовательно, и приведенная производящая функция) определяются ростком пары неоднозначно.

Выбор таких координат Дарбу равносилен выбору ростка симплектоморфизма, переводящего росток многообразия $C$ в росток координатного коизотропного подпространства и касательное пространство к $L$ в нуле в координатное лагранжево подпространство $p=0$.

Таким образом, множества приведенных производящих функций у эквивалентных пар совпадают.

Обозначим через $G_{k}$ группу ростков в нуле симплектоморфизмов стандартного симплектического пространства $\mathbb{R}^{2 n}$, сохраняющих коизотропное подпространство $C_{0}=\left\{p_{1}=\cdots=p_{k}=0\right\}$ и касательное пространство в нуле к лагранжеву подмногообразию $L_{0}=\{p=0\}$, а через $G_{k}^{+}$- eе подгруппу, элементы которой сохраняют ориентацию пространства $C_{0}$. 3aметим, что $G_{k}$ изоморфна полупрямому произведению подгруппы $G_{k}^{+}$и группы $\mathbb{Z}_{2}$, порожденной отражением

$$
I:\left(p_{1}, p_{2}, \ldots, p_{n}, q_{1}, q_{2}, \ldots, q_{n}\right) \mapsto\left(-p_{1}, p_{2}, \ldots, p_{n},-q_{1}, q_{2}, \ldots, q_{n}\right),
$$

меняющим ориентацию подпространства $C_{0}$.

Итак, классификация эквивалентных пар с точностью до надстройки сводится к классификации приведенных производящих функций относительно действия $G_{k}$ на графиках их градиентов.

Пусть $k<n$. Обозначим через $Q\left(z_{1}, \ldots, z_{k}\right)$ невырожденную квадратичную форму $Q= \pm z_{1}^{2} \pm \cdots \pm z_{k}^{2}$.

ТЕОРемА 1. (i) Всякий простой росток эквивалентен надстройке над ростком пары $\left(L^{n}, C^{2 n-1}\right)$, в которой $C^{2 n-1}$ - гиперповерхность.

(ii) Всякий простой росток $\left(L^{n}, C^{2 n-1}\right)$ нетрансверсального пересечения $L$ и $C$ эквивалентен ростку пары $\left(L_{S}^{n}, C_{0}^{2 n-1}\right)$, заданному в координатах Дарбу $\left(y, p_{1}, \ldots, p_{n-1} ; x, q_{1}, \ldots, q_{n-1}\right), \omega=d y \wedge d x+\sum_{i=1}^{n-1} d p_{i} \wedge d q_{i}$, гunepnoверхностью $C_{0}=\{y=0\}$ и лагранжевым подмногообразием $L_{S}$, имеюиим производящую функиию $S\left(x, q_{1}, \ldots, q_{n-1}\right)$ одного из следующих видов:

1) $S=x^{3}+x f(q)$, где $f(q) \in \mathbf{m}_{q}^{2}$ - одна из простых особенностей Фнкиий

$A_{k}: k \geqslant 2, f(q)= \pm q_{1}^{k+1}+Q\left(q_{2}, \ldots, q_{n-1}\right)$

(при нечетном $k$ особенности со знаками \pm эквивалентнь),

$D_{k+1}: k \geqslant 3, f(q)=q_{1}^{2} q_{2} \pm q_{2}^{k}+Q\left(q_{3}, \ldots, q_{n-1}\right)$,

$E_{6}: f(q)=q_{1}^{3} \pm q_{2}^{4}+Q\left(q_{3}, \ldots, q_{n-1}\right)$,

$E_{7}: f(q)=q_{1}^{3}+q_{1} q_{2}^{3}+Q\left(q_{3}, \ldots, q_{n-1}\right)$,

$E_{8}: f(q)=q_{1}^{3}+q_{2}^{5}+Q\left(q_{3}, \ldots, q_{n-1}\right)$,

2) $C_{k}: k \geqslant 2, S=x^{2 k+1}+x^{2} q_{1}+x Q\left(q_{2}, \ldots, q_{n-1}\right)$,

3) $B_{k}: k \geqslant 2, S=x^{3} q_{2}+x^{2} q_{1}+x\left( \pm q_{2}^{k}+Q\left(q_{3}, \ldots, q_{n-1}\right)\right)$,

4) $F_{4}: S=x^{5}+x^{2} q_{1}+x\left(q_{2}^{3}+Q\left(q_{3}, \ldots, q_{n-1}\right)\right)$,

5) $U_{3}: S= \pm x^{4}+x Q\left(q_{1}, \ldots, q_{n-1}\right)$. 
ЗАмЕЧАНИЕ 1. 1. Классы $C_{k}, F_{4}$ имеют другую эквивалентную форму (при $n>2$ ): $S=x^{3} q_{2}+x^{2} q_{1}+x f$, где $f-$ соответствуюшая простая краевая особенность функции от $q_{2}, \ldots, q_{n-1}$ с краем $q_{2}=0$. В частности, $B_{2}$ эквивалентно $C_{2}$.

2. Таблица примыканий отличается от известной для краевых особенностей лишь примыканием $U_{3} \rightarrow B_{2}$.

Для особенности $A_{1}$ пары справедливо более сильное утверждение.

Теорема 2 (симплектическая лемма Морса). Пусть $h:\left(\mathbb{R}^{2 n}, 0\right) \rightarrow(\mathbb{R}, 0)$ - росток регулярной функции и $(L, 0)$ - такой росток в нуле лагранжева подмногообразия, что ограничение $h$ на $L$ имеет в нуле невырожденную (морсовскую) критическую точку. Пусть выполнено следуюшее условие обшности положения: характеристическое направление аиперповерхности $h=0$ не принадлежит касательному конусу $\kappa$ пересечению $L \cap\{h=0\}$.

Тогда симплектоморфизмом некоторой окрестности нуля в $\mathbb{R}^{2 n}$ и гладкой заменой в пространстве-образе функиии $h$ росток в нуле парьи $L u$ $h$ приводится $\kappa$ паре, состоящей из функиии $h=y$ и лагранжева многообразия, имеющего производящую функиию $S=x^{3}+x\left( \pm q_{1}^{2} \pm \cdots \pm q_{n-1}^{2}\right)$ в некоторых координатах Дарбу $y, p_{1}, \ldots, p_{n-1}, x, q_{1}, \ldots, q_{n-1}$.

СЛЕДСТВИЕ 1. Если гиперповерхность уровня $h=0$ обшим образом касается в нуле лагранжева многообразия $L^{n}$, то в семействе редучированньх лагранжевых подмногообразий $l_{c}=\rho(L,\{h=c\})$, лежаших в симплектических многообразиях характеристик гиперповерхностей $h=c$, при прохождении значения с через 0 рождается ияи умирает одна точка трансверсального самопересечения.

Пусть на (конфигурационном) многообразии $M^{n}$ задана натуральная механическая (гамильтонова) система. То есть заданы кинетическая энергия $T: T^{*} M \rightarrow \mathbb{R}$, являющаяся положительно определенной квадратичной формой на слоях, и потенциальная энергия $\Pi: M \rightarrow \mathbb{R}$. Пусть при всяком значении гамильтониана $h=T+\Pi$ из некоторого интервала пространство характеристик (траекторий) системы изоморфно некоторому гладкому симплектическому многообразию.

Рассмотрим траектории системы, начальные условия которых принадлежат лагранжеву подмногообразию ковекторов $T^{*} M$, обращающихся в нуль на касательных пространствах к заданному гладкому стартовому подмногообразию $\widetilde{M}$ общего положения в конфигурационном пространстве.

СлЕДСТВИЕ 2. Четность числа траекторий, достигающих границь области возможности движения (m.е. пересечения гиперповерхности уровня гамильтониана с нулевьм сечением кокасательного расслоения), равна четности числа критических точек ограничения функиии П на $\widetilde{M}$.

Доказательство следствия 2 вытекает из теоремы 2 и свойства обратимости натуральных механических систем.

ЗАмЕчАНИЕ 2 . Простые пары в случае, когда $k=n$, т. е. $C-$ лагранжево подмногообразие, описываются известной теоремой Голубицкого-Гийемина о контакте лагранжевых подмногообразий. Простые особенности здесь отвечают особенностям $A, D, E$ производящих функций (см. также замечание 3 ниже). 


\section{Доказательства}

Нам потребуются следующие элементарные свойства рассматриваемой группы преобразований.

ЛЕмма 1. Симплектоморфизм $\varphi$ из $G_{k}^{+}$можно соединить гладкой гомотопией в $G_{k}^{+}$с тождественньм преобразованием.

ДоказАТЕльство. Линейные части $\varphi_{*}$ в нуле симплектоморфизмов $\varphi$ из $G_{k}^{+}$, сохраняющих $C_{0}$ и $L_{0}$, образуют, как легко видеть, связную подгруппу $\Lambda_{k}$ симплектической линейной группы. Симплектоморфизм же $\widetilde{\varphi}=\left(\varphi_{*}^{-1}\right) \varphi$ имеет в нуле единичную линейную часть, т. е. достаточно близок к тождественному вблизи нуля. Поэтому локально он задается «производящей» функцией $\mathscr{F}(P, q)$ (с нулевой 2-струей в нуле) по формулам

$$
\widetilde{\varphi}:(p, q) \mapsto(P, Q), \quad \sum_{i=1}^{n}\left(Q_{i} d P_{i}+p_{i} d q_{i}\right)=d\left(\sum_{i=1}^{n} P_{i} q_{i}+\mathscr{F}(P, q)\right) .
$$

Условие сохранения $C_{0}$ означает, что функции

$$
p_{i}=P_{i}+\frac{\partial \mathscr{F}}{\partial q_{i}}
$$

при $i=1, \ldots, k$ обращаются в нуль, если $P_{1}=\cdots=P_{k}=0$. Оно, очевидно, выполнено и для семейства симплектоморфизмов $\tilde{\psi}_{t}$ с производящими функциями $t \mathscr{F}(P, q), t \in[0,1]$. Искомая гомотопия является композицией симплектоморфизма $\tilde{\psi}_{t}$ и соответствующей гомотопии в $\Lambda_{k}$.

ЛЕмма 2. Если гамильтоново векторное поле $V_{h}$ касается коизотропного подмногообразия $C$, то его гамильтониан $h$ постолнен на всяком слое характеристического слоения этого подмногообразия.

ДокАЗАТЕльСТво. Подмногообразие $C$ является совместным множеством нулей некоторых независимых регулярных функций $f_{1}, \ldots, f_{k}$, находящихся в инволюции. Коммутирующие (в точках из $C$ ) гамильтоновы векторные поля $V_{f_{i}}$ порождают касательные пространства к слоям характеристического слоения.

Условие касания поля $V_{h}$ подмногообразия $C$ означает, что $L_{V_{h}} f_{i}=0$, но $L_{V_{h}} f_{i}=\left[h, f_{i}\right]=-L_{V_{f_{i}}} h$. Таким образом $h$ постоянна вдоль всякого $V_{f_{i}}$, т. е. вдоль всякого слоя.

Применяя эту лемму к координатному подпространству $C_{0}$, получаем

СлЕДСТВИЕ 3. Инфинитезимальные преобразования из $G_{k}^{+}$задаются гамильтоновьми векторными полями с гамильтонианами $h$, представимьми в виде

$$
h=\sum_{i=1}^{k} p_{i} h_{i}(p, q)+h_{0}\left(p_{k+1}, \ldots, p_{n}, q_{k+1}, \ldots, q_{n}\right)
$$

c некоторыми гладкими $h_{i} u h_{0}$. Гамильтониан $h_{0}$ определяет редуцированное векторное поле на базе характеристического расслоения.

Лемма 3. Пусть в некоторых координатах Дарбу $p$, q росток лагранжева многообразия $L \subset \mathbb{R}^{2 n}$ задается производящей функцией $S(q)$, и пусть $\Phi_{t}$ - однопараметрическое семейство локальньх симплектоморфизмов, заданное гамильтоновым Фазовым потоком с гамильтонианом $H_{t}(p, q)$. То- 
гда производящие семейства $S_{t}(q)$ (в этих же координатах) лагранжевых многообразий $\Phi_{t}(L)$ удовлетворлют условию

$$
\frac{\partial S_{t}}{\partial t}=-H_{t}\left(\frac{\partial S_{t}}{\partial q}, q\right)
$$

ДокАЗАТЕЛЬСТво. Лагранжевы подмногообразия $\Phi_{t}(L)$, вложенные каждое в свой временной слой $t$ расширенного фазового пространства $T^{*} \mathbb{R}^{n+1}$, образуют лагранжево подмногообразие с производящей функцией $S(t, q)=$ $S_{t}(q)$ на $\mathbb{R}^{n+1}$, которая удовлетворяет неавтономному уравнению Гамильтона-Якоби $\partial S / \partial t+H_{t}(\partial S / \partial q, q)=0$, что и требовалось показать.

Координаты $q_{i}$ будем далее обозначать через $x_{i}$ при $i=1, \ldots, k$ и через $z_{i-k}$ при $i=k+1, \ldots, n$, а двойственные координаты $p_{i}-$ соответственно через $y_{i}$ и $w_{i-k}$.

Итак, касательное пространство $T_{S} G$ к $G_{k}^{+}$-орбите приведенной функции $S$ по лемме 3 и следствию 3 состоит из всех функций вида

$$
\sum_{i=1}^{k} \frac{\partial S}{\partial x_{i}} h_{i}\left(\frac{\partial S}{\partial x}, \frac{\partial S}{\partial z}, x, z\right)+h_{0}\left(\frac{\partial S}{\partial z}, z\right)
$$

с гладкими $h_{i}$ и $h_{0}$, причем $h_{i}(0)=0, h_{0}(w, z) \in \mathbf{m}_{w, z}^{2}$.

Группа $G_{k}^{+}$содержит подгруппу $R_{k}^{+}$симплектоморфизмов, сохраняющих еще и лагранжево расслоение $(p, q) \mapsto q$. Преобразования из $R_{k}^{+}$действуют на производящие функции $S$ как правые замены переменных $q$, расслоенные над пространством $z$, и сложения с функциями от $z$ (с нулевой 2-струей).

Касательное пространство $T_{S} R^{+}$к орбите этой группы отличается от предыдущего тем, что функции $h_{0}$ аффинны по $\partial S / \partial z$.

СлЕДСТвиЕ 4. Если графики градиентов $L\left(S_{1}\right)$ и $L\left(S_{2}\right)$ приведенных функиий $S_{1}$ и $S_{2} G_{k}^{+}$-эквивалентнь, то 3 -струи $j^{3} S_{i}$ этих функций $R_{k}^{+}$-эквивалентны и, в частности, 3-струи сужений этих функций на слой $z=0$ правоэквивалентны.

ДокАЗАТЕЛЬСТво. Воспользуемся леммой 1 и выберем переводящую $L\left(S_{1}\right)$ в $L\left(S_{2}\right)$ гомотопию в $G_{k}^{+}$, являющуюся фазовым потоком с некоторым гамильтонианом $h_{t}$, удовлетворяющим при всяком $t$ условию следствия 3 . Поскольку начало координат все время остается на месте, значение $h_{0}$ в начале координат можно считать нулевым. В силу леммы 3 соответствуюшее семейство производящих функций $S_{t}$ удовлетворяет условию

$$
\frac{\partial S_{t}}{\partial t}=-\sum_{i=1}^{k} \frac{\partial S_{t}}{\partial x_{i}} h_{i t}\left(\frac{\partial S_{t}}{\partial q}, q\right)+h_{0 t}\left(\frac{\partial S_{t}}{\partial z}, z\right) \text {. }
$$

Для приведенной производящей функции $S$ 3-струи в нуле касательных пространств $J^{3} T_{S} G_{k}$ и $J^{3} T_{S} R_{k}^{+}$совпадают. Действительно, эти пространства различаются множеством функций, принадлежащих квадрату идеала, порожденного функциями $\partial S / \partial z$, каждая из которых принадлежит $\mathbf{m}_{q}^{2}$. Итак, $j^{3} \partial S_{t} / \partial t \in J^{3} T_{S} R_{k}^{+}$. В частности, положив $z=0$ и введя обозначение 
$s_{t}(x)=S_{t}(x, 0)$, получаем

$$
\frac{\partial s_{t}}{\partial t}=-\sum_{i=1}^{k} \frac{\partial s_{t}}{\partial x_{i}} \tilde{h}_{i}(x, t) \bmod \left\{\mathbf{m}_{x}^{4}\right\}
$$

с некоторыми гладкими $\tilde{h}_{i}$. Тем самым выполнены соответствуюшие гомологические уравнения для семейства $j^{3} S_{t}$ (по отношению к группе $R_{k}^{+}$) и для 3 -струи семейства $s_{t}$ (по отношению к группе правых замен), что и требовалось.

ЗАмечАниЕ 3. 1. Орбите всей группы $G_{k}$ также отвечает правая орбита $j^{3} s$, поскольку отражение $I$ действует на производящую функцию правой заменой $\left(q_{1}, q_{2}, \ldots, q_{n}\right) \mapsto\left(-q_{1}, q_{2}, \ldots, q_{n}\right)$.

2 (теорема Голубицкого-Гийемина). Если $k=n$, то $G_{k}$-орбиты касающихся лагранжевых $L$ и $C$ классифицируются правыми орбитами $S$ (в этом случае $\left.T_{S} G_{k}=T_{S} R_{k}^{+}\right)$.

ДоКАЗАТЕЛЬСТво ТЕОРемы 1 . Простым $G_{k}$-орбитам отвечают, очевидно, простые правые орбиты 3-струй сужений функций $s(x)$. Приведенным функциям $S(q)$ отвечают сужения из $\mathbf{m}_{x}^{3}, 3$-струи которых не просты при $k>2$.

Все особенности с $k=2$ примыкают к классу $D_{4}^{ \pm}$с $j^{3} s_{D}(x)=x_{1}^{3} \pm x_{1} x_{2}^{2}$. Всякая функция $S$ из этого класса $R_{2}^{+}$-эквивалентна функции со следующей 3-струей:

$$
j^{3} S(x, z)=s_{D}(x)+\lambda_{1}(z)\left(x_{1}^{2} \mp x_{2}^{2}\right)+\lambda_{2}(z) x_{1}+\lambda_{3}(z) x_{2} .
$$

При этом линейная форма $\lambda_{1}$ и квадратичные формы $\lambda_{2}, \lambda_{3}$ определены $G_{2}$-орбитой функции $S$ с точностью до правой замены.

Такой набор, конечно, имеет непрерывные инварианты (в количестве $2(n-k))$ относительно группы линейных замен $z$. Итак, простых пар с $k \geqslant 2$ не существует. Пункт (i) теоремы 1 доказан.

Осталось рассмотреть случай $k=1$. В дальнейшем индекс 1 у $G_{1}$ и $R_{1}^{+}$ будем опускать. В пространстве 3 -струй семейств функций одной переменной $x$ с параметрами $z \in \mathbb{R}^{n-1} R^{+}$-орбиты разбиваются на три класса

$$
x^{3}+x \widetilde{Q}(z) \leftarrow x^{2} z_{1}+x \widetilde{Q}_{2}\left(z_{2}, \ldots, z_{n-1}\right) \leftarrow x \widetilde{Q}(z) .
$$

Здесь $\widetilde{Q}(z), \widetilde{Q}_{2}\left(z_{2}, \ldots, z_{n-1}\right)$ - представители орбит действия линейной группы на квадратичных формах от $z$ и $z_{2}, \ldots, z_{n-1}$ соответственно.

Функция $S$ из первого такого класса $R^{+}$-эквивалентна функции вида $S(q)=x^{3}+x \lambda(z)$. Касательное пространство к ее $G$-орбите

$$
T_{S} G=\left\{\left(3 x^{2}+\lambda(z)\right) h(x, z)+h_{0}\left(x \frac{\partial \lambda}{\partial z}, z\right)\right\}
$$

совпадает для такой функции с касательным пространством к ее $R^{+}$-орбите

$$
T_{S} R^{+}=\left\{\left(3 x^{2}+\lambda(z)\right) h(x, z)+x \frac{\partial \lambda}{\partial z} a(z)+b(z)\right\} .
$$

Здесь $h, a, b$ - произвольные гладкие функции. Таким образом, $G$-орбиты таких функций $S$ находятся во взаимно однозначном соответствии с правыми орбитами функций $\lambda(z)$, что и доказывает п. (ii) теоремы 1. 
Рассмотрение оставшихся случаев основано на осторожном (поскольку касательное пространство к $G$-орбитам зависит от $S$ нелинейно) применении спектральной последовательности Арнольда [7].

Гамильтониан $H$ инфинитезимального преобразования из группы $G$ принадлежит $\mathbf{m}_{p, q}^{2}$; поэтому $m$-струя $J^{m} T_{S} G$ касательного пространства $T_{S} G$ к $G$-орбите функции $S$ зависит только от $j^{m} S$. Пусть функции $S_{0}$ и $S_{1}$ соединены гомотопией $S_{t}, t \in[0,1]$, принадлежащей одной орбите группы $G$; тогда $m$-струи $s_{t}$ функций $S_{t}$ удовлетворяют соотношению

$$
\frac{\partial s_{t}}{\partial t}=-j^{m}\left(H_{t}\left(\frac{\partial s_{t}}{\partial q}, q\right)\right),
$$

которое можно рассматривать как обыкновенное дифференциальное уравнение относительно компонент $m$-струй $s_{t}$. Коэффициенты полиномиальной правой части этого уравнения определяются струей соответствующего семейства гладких гамильтонианов $H_{t}$. В силу единственности решения $s_{t}$ такого уравнения (при фиксированном $H_{t}$ ) с заданным начальным условием $s_{0}$ семейство $G$-эквивалентностей, порожденное семейством гамильтонианов $H_{t}$, переводит произвольное продолжение $\widetilde{S}$ струи $s$ в семейство функций $\widetilde{S}_{t}, m$-струи которых при всяком $t$ совпадают с $s_{t}$.

Две $m$-струи $s_{0}$ и $s_{1}$ назовем $G_{m}^{+}$-эквивалентныли, если они соединяются гомотопией $s_{t}$, для которой $\partial s_{t} / \partial t \in J^{m} T_{S_{t}} G$, т. е. гомотопией, которая является решением (с заданными граничными условиями) указанного обыкновенного дифференциального уравнения, отвечающего некоторому семейству $m$-струй гамильтонианов $H_{t}$. Струи $s_{0}$ и $s_{1}$ будем называть $G_{m}$-эквивалентныли (и обозначать это так: $s_{0} \approx s_{1}$ ), если $s_{0} G_{m}$-эквивалентна либо $s_{1}$, либо $I\left(s_{1}\right)=S_{1}(-x, z)$. Ясно, что $j^{m}$-струи функций из одной $G$-орбиты принадлежат одному классу $\approx$-эквивалентности.

ЛЕмма 4 (определитель особенностей с вырожденными 3-струями).

1. Пусть 3-струя приведенной производящей функиии $S$ принадлежит второму классу: $j^{3} S \approx x^{2} z_{1}+x \widehat{Q}\left(z_{2}, \ldots, z_{n-1}\right)$.

1.0. Если $\widehat{Q}(z)$ невьгрожденна, то $j^{4} S \approx x^{2} z_{1}+x\left( \pm z_{2}^{2} \pm \cdots \pm z_{n-1}^{2}\right)$ и либо $J^{5} S \approx x^{5}+x^{2} z_{1}+x \widehat{Q}$ (в этом случае функция $S$ G-эквивалентна нормальной борме $\left.C_{2}\right)$, либо найдется такое $k>2$, ито $j^{i}(S) \approx x^{2} z_{1}+x \widehat{Q}$ при всех $i \leqslant 2 k$ u $j^{2 k+1} S \approx x^{2 k+1}+x^{2} z_{1}+x \widehat{Q}$ (в этом случае $S$ G-эквивалентна нормальной форме $C_{k}$ ), либо $S$ имеет коразмерность $\infty$ в пространстве фнкиий.

1.1. Если коранг формы $\widehat{Q}$ равен $1, \widehat{Q}= \pm z_{3}^{2} \pm \cdots \pm z_{n-1}^{2}$, то либо $j^{4} S \approx$ $x^{3} z_{2}+x^{2} z_{1}+x \widehat{Q}($ тогда функиия $S$ либо $G$-эквивалентна одной из нормальных форм $B_{k}$, либо принадлежит подмножеству коразмерности $\left.\infty\right)$, либо $j^{5} S \approx x^{5}+x^{2} z_{1}+x\left(z_{2}^{3} \pm z_{3}^{2} \pm \cdots \pm z_{n-1}^{2}\right)$ (и в этом случае $S$ G-эквивалентна $\left.F_{4}\right)$, либо $j^{5} S \approx x^{2} z_{1}+x\left(z_{2}^{3} \pm z_{3}^{2} \pm \cdots \pm z_{n-1}^{2}\right)$ и тогда $j^{6} S \approx x^{2} z_{1}+\cdots+z_{2} x^{5} a$ $(a \in \mathbb{R}-$ модуль $)$, либо $j^{5} S \approx x^{5}+x^{2} z_{1}+x\left(z_{2}^{4} \pm z_{3}^{2} \pm \cdots \pm z_{n-1}^{2}\right)=a x^{3} z_{2}^{2}$

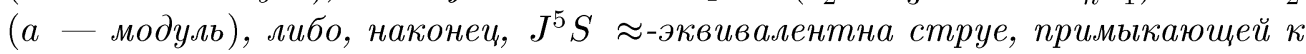
предьдущему классу (и, таким образом, также не проста).

1.2. Если коранг формыл $\widehat{Q}$ равен $2, \widehat{Q}= \pm z_{4}^{2} \pm \cdots \pm z_{n-1}^{2}$, то либо $j^{4} S \approx$ 
$x^{3} z_{2}+x^{2} z_{1}+x\left(\widehat{Q}+z_{3}^{3}+a z_{3} z_{2}^{2}+z_{3}^{3}\right)(a-$ модуль $)$, либо $j^{4} S \approx x^{2} z_{1}+x\left(\widehat{Q}+z_{2}^{3} \pm\right.$ $\left.z_{2} z_{3}^{2}\right)$ или $j^{4} S$ примькает $\kappa$ этому классу. В этих случаях $j^{5} S$ не проста.

При коранге формы $\widehat{Q}$, большем 2, 5-струи также не простьл.

2. Пусть 3-струя функиии $S$ принадлежит третьему классу $j^{3} S \approx x \widehat{Q}$.

2.0. Если $Q$ невырожденна, то либо $j^{4} S \approx x^{4}+x \widehat{Q}$ (и тогда $S$ G-эквивалентна особенности $\left.U_{3}\right)$, либо $j^{4} S \approx x^{3} l(z)+x \widehat{Q}$, где $l(z)$ - линейная форма от z, коэффициенть которой являются модулями.

2.1. Если $Q$ вырожденна, то $j^{4} S \approx x^{4}+a x^{2} z_{2}^{2}+x\left(z_{2}^{3} \pm z_{3}^{2} \pm \cdots \pm z_{n-1}^{2}\right)$ (а - модуль) или $j^{4} S$ примыкает $к$ этому классу.

ДокАЗАТЕЛЬСтво ЛЕммы 4 . Предположим, что $m$-струя функции $S$ преобразованием из группы $G_{k}$ приведена к нормальной форме $s_{m}$; тогда $S$ эквивалентна некоторому продолжению $s_{m}$. На $(m+1)$-струи таких продолжений будем действовать подгруппой $G_{k+1}^{s t} G_{k+1}$-преобразований, сохраняющих $s_{m}$.

Касательное пространство $K^{k+1}\left(s_{m+1}\right)$ к $G_{k+1}^{s t}$-орбите этой подгруппы содержит только такие струи, компоненты которых аффинно зависят от компонент степени $m+1$ продолжений $s_{m+1}$. Таким образом, $G_{k+1}^{s t}$-орбиты в пространстве однородных функций степени $m+1$ совпадают с орбитами действия некоторой подгруппы аффинной группы этого пространства. Представители таких орбит являются $G_{k+1}$-нормальными формами $s_{m+1}$.

Простые орбиты примыкают не более чем к конечному числу других орбит; поэтому если для всех продолжений коразмерность $K^{k+1}\left(s_{m+1}\right)$ в пространстве однородных функций степени $m+1$ положительна, то классы всех $s_{m+1}$ не просты.

Применение этого спектрального метода индукцией по $m$ для всех возникающих $G_{k}$-простых орбит и приводит к искомой классификации.

Например, в случае 1.0 пространство $K^{4}\left(s_{4}\right)$ всякого 4-продолжения струи $j^{3} S=x^{2} z_{1}+x \widehat{Q}\left(z_{2}, \ldots, z_{n-1}\right)$ содержит 4-струи следующих функций (здесь $\sim$ означает равенство по модулю $\mathbf{m}_{x, z}^{5}$ )

$$
\begin{array}{rlrl}
\frac{\partial S}{\partial z_{1}} z_{i} z_{j} \sim x^{2} z_{i} z_{j}, & \left(\frac{\partial S}{\partial z_{1}}\right)^{2} & \sim x^{4}, & \frac{\partial S}{\partial z_{1}} \frac{\partial S}{\partial z_{j}} \sim 2 x^{3} z_{j}, \\
\frac{\partial S}{\partial z_{j}} z_{i} z_{k} \sim 2 x z_{i} z_{j} z_{k}, & \frac{\partial S}{\partial z_{j}} \frac{\partial S}{\partial z_{m}} & \sim 4 x^{2} z_{j} z_{m}, \\
\frac{\partial S}{\partial x} z_{i} z_{j} \sim\left(2 x z_{1}+Q\right) z_{i} z_{j}, & \frac{\partial S}{\partial x} x^{2} & \sim 2 x^{3} z_{1}+x^{2} Q,
\end{array}
$$

а также все мономы 4-й степени от z. Таким образом, это касательное пространство содержит пространство всех 4-продолжений, которые, следовательно, образуют одну орбиту.

Итак, $G$-эквивалентностью приведем функцию $S$ к виду $S_{(5)}$, для которого 4-струя нормализована: $j^{4} S_{(5)}=x^{2} z_{1}+x \widehat{Q}$. Если $j^{5} S_{(5)}$ содержит с ненулевым коэффициентом моном $x^{5}$, то, как легко видеть, классы перечисленных выше образующих, а также функций $\left(\partial S_{(5)} / \partial z_{1}\right)^{3},\left(\partial S_{(5)} / \partial x\right) x$, порождают над кольцом $C_{z}$ (ростков функций от $z$ ) фактор модуля $\mathbf{m}_{x, z}^{5}$ по 
идеалу, порожденному над $C_{x, z}$ функцией $(\partial S / \partial x) x^{3}$ и $\mathbf{m}_{z}^{6}$. По лемме Накаямы классы этих функций порождают и фактор $\mathbf{m}_{x, z}^{5} / C_{x, z}\left\{(\partial S / \partial x) x^{3}\right\}$, т.е. $\mathbf{m}_{x, z}^{5}$ принадлежит $T_{S_{(5)}} G$. Итак, 5-струя является достаточной, т.е. гомотопическим методом функция $S_{(5)}$ приводится к виду $C_{2}$.

Если же в $j^{5} S_{(5)}$ коэффициент при $x^{5}$ нулевой, то $J^{5} T_{S_{(5)}} G$ имеет положительную коразмерность в пространстве 5 -струй (не содержит класса $x^{5}$ ). Таким образом, этот случай не $G$-эквивалентен предыдущему (он инвариантен по отношению к $G_{5}^{s t}$-преобразованиям, сохраняющим 4-струю $\left.S_{(5)}\right)$. Все такие 5-струи образуют одну $G_{5}^{s t}$-орбиту. Подобно случаю 4 -струи, легко видеть, что все 6 -продолжения 5 -струи $j^{5} S=x^{2} z_{1}+x \widehat{Q}$ эквивалентны между собой. Рассмотрение старших струй приводит поочередно к классам $C_{k}$ или к классу коразмерности $\infty$ (примыкающему ко всем $C_{k}$ ). Остальные пункты определителя особенностей рассматриваются аналогично.

ДокАЗАТЕЛЬСТво теоремы 2. В некоторых координатах Дарбу $(p, q)=$ $(y, w, x, z), \omega=d y \wedge d x+\sum_{i=1}^{n-1} d w_{i} \wedge d z_{i}$, расслоение на гиперповерхности уровня функции $\varphi$ выпрямляется: $\varphi=\varphi(y)$, и лагранжево многообразие $L$ задается приведенной производящей функцией $S(x, z)$.

Группе $G_{y}$ симплектоморфизмов, сохраняющих такое расслоение, отвечает алгебра гамильтоновых векторных полей, $\partial / \partial y$-компонента которых зависит только от $y$. Их гамильтонианы представляются в виде

$$
H=x h_{1}(y)+h_{2}(y, z, w) .
$$

Квадратичность касания $L$ и гиперповерхности $\{y=0\}$, а также и условие невырожденности означают, что функция $S$ приводится $R^{+}$-эквивалентностью (с сохранением только слоя $\{y=0\})$ к функции $S_{0}=x^{3}+x\left( \pm z_{1}^{2} \pm\right.$ $\left.\cdots \pm z_{n-1}^{2}\right)$ класса $A_{1}$. При этом 3 -струю $j^{3} S$ можно привести к такому виду линейным симплектоморфизмом из $R^{+} \subset G$ и сдвигом, порожденным гамильтонианом, зависящим только от $z$. Такие преобразования, очевидно, принадлежат и группе $G_{y}$.

Пользуясь стандартным гомотопическим методом, для доказательства теоремы достаточно показать, что касательное пространство к орбите группы $G_{y}$ в каждом продолжении

$$
S=S_{0}+S_{t}, \quad S_{t} \in \mathbf{m}_{p, q}^{4}(t),
$$

этой 3-струи содержит все пространство $\mathbf{m}_{p, q}^{4}(t)$, состоящее из функций от $p, q$ и дополнительного параметра $t$, при каждом $t$ принадлежащих $\mathbf{m}_{p, q}^{4}$. По лемме 3 такое касательное пространство есть множество сужений гамильтониана $H$ на лагранжево подмногообразие $L(S)$ :

$$
y=3 x^{2} \pm z_{1}^{2} \pm \cdots \pm z_{n-1}^{2}+\frac{\partial S_{t}}{\partial x}, \quad w_{i}= \pm 2 x z_{i}+\frac{\partial S_{t}}{\partial z_{i}}, \quad i=1, \ldots, n-1 .
$$

Рассмотрим произвольную функцию $\beta(x, z, t)$. В силу подготовительной теоремы Мальгранжа (примененной к отображению $(x, z, t) \mapsto(y, z, t))$ существует разложение

$$
\beta=x H_{1}+H_{0},
$$


где $H_{0}, H_{1}$ - гладкие функции от $y, z, t$. Обозначим через $B_{i 1}(y, z, t)$, $B_{i 0}(y, z, t)$ коэффициенты подобных разложений для функций $\partial S_{t} / \partial z_{i}$ :

$$
\frac{\partial S_{t}}{\partial z_{i}}=x B_{i 1}+B_{i 0} .
$$

Заметим, что $B_{i j} \in \mathbf{m}_{y, z}^{2}$ при любом $t$; поэтому по теореме деления функцию $H_{1}(y, z, t)$ можно представить в виде

$$
H_{1}(y, z, t)=h_{0}(y, t)+\sum_{i=1}^{n-1}\left( \pm 2 z_{i}+B_{i 1}\right) h_{i 1}(y, z, t) .
$$

Подставляя это выражение в формулу разложения для $\beta$, получаем

$$
\begin{aligned}
\beta & =x h_{0}+\sum_{i=1}^{n-1}\left( \pm 2 x z_{i}+x B_{i 1}\right) h_{i 1}+H_{0} \\
& =x h_{0}+\sum_{i=1}^{n-1}\left( \pm 2 x z_{i}+x B_{i 1}+B_{i 0}\right) h_{i 1}+H_{0}-\sum_{i=1}^{n-1} B_{i 0} h_{i 1} .
\end{aligned}
$$

Заметим, что выражения в скобках равны $w_{i}$; таким образом,

$$
\beta=x h_{0}(y, t)+\sum_{i=1}^{n-1} w_{i} h_{i 1}(y, z, t)+\tilde{h}(y, z, t),
$$

где $\tilde{h}=H_{0}-\sum_{i=1}^{n-1} B_{i 0} h_{i 1}$. Это равенство и является искомым представлением функции $\beta(x, z, t)$ в качестве элемента касательного пространства к $G_{y}$-орбите. Теорема 2 доказана.

ЗАмЕчАниЕ 4. Фактически мы доказали большее. Найденный гамильтониан является аффинным по переменным $w_{i}$, т.е. $L\left(S_{0}+S_{t}\right)$ можно привести к $L\left(S_{0}\right)$ симплектоморфизмом, сохраняющим лагранжево расслоение $(y, w, x, z) \mapsto(y, z)$.

\section{ЛИтеРАТУРА}

1. Арнольд В. И. Лагранжевы многообразия с особенностями, асимптотические лучи и раскрытый ласточкин хвост. Функц. анализ и его прил., 15, вып. 4, $1-14$ (1981).

2. Щербак О. П. Волновые фронты и группы отражений. УМН, 43, вып. 3, 125160 (1988).

3. Гивенталь A. Б. Особые лагранжевы многообразия и их лагранжевы отображения. В кн.: Современные проблемы математики. Новейшие достижения. Т. 33, ВИНИТИ, М., 1988, с. 55-112.

4. Закалюкин В. М. Одно обобщение лагранжевых триад. УМН, 41, вып. 4, 180 (1986).

5. Закалюкин В. М., Робертс Р. М. Об устойчивых лагранжевых многообразиях с особенностями. Функц. анализ и его прил., 26, вып. 3, 28-34 (1992).

6. Domitrz W., Janeczko S. Equivalence of Lagrangian germs in the presence of a surface. Banach Center Publ., Vol. 39, pp. 31-37.

7. Арнольд В. И., Варченко А. Н., Гусейн-Заде С. М. Особенности дифференцируемых отображений 1. Наука, М., 1982. 